ARTIGO ORIGINAL

\title{
Extração de óleo de andiroba por prensa: rendimento e qualidade de óleo de sementes submetidas a diferentes teores de água e temperaturas de secagem
}

\author{
Extraction of andiroba oil by press: yield and quality of seed oil submitted \\ to different moisture and drying temperatures
}

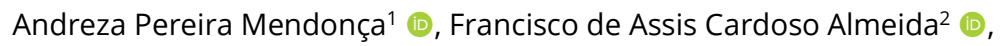

Angélica dos Santos Oliveira ${ }^{1}$ (i) , Jhonatas Cortes Rosa ${ }^{3}$ (i), Maria Elessandra Rodrigues Araújo ${ }^{1}$ (D), Paulo de Tarso Barbosa Sampaio ${ }^{4}$ (1)

${ }^{1}$ Instituto Federal de Educação Ciência e Tecnologia de Rondônia - IFRO, Ji-Paraná, RO, Brasil

¿Universidade Federal de Campina Grande - UFCG, Campina Grande, PB, Brasil

${ }^{3}$ Fundação Universidade Federal de Rondônia - UNIR, Rolim de oura, RO, Brasil

${ }^{4}$ Instituto Nacional de Pesquisas da Amazônia - INPA, Manaus, AM, Brasil

Como citar: Mendonça, A. P., Almeida, F. A. C., Oliveira, A. S., Rosa, J. C., Araújo, M. E. R., \& Sampaio, P. T. B. (2020). Extração de óleo de andiroba por prensa: rendimento e qualidade de óleo de sementes submetidas a diferentes teores de água e temperaturas de secagem. Scientia Forestalis, 48(125), e2995.

https://doi.org/10.18671/scifor.v48n125.09

\begin{abstract}
Resumo
As sementes de andiroba (Carapa surinamensis e Carapa guianensis) foram coletadas em plantios de propriedades particulares em ambiente de terra firme na zona rural em Rondônia, Brasil. O objetivo do trabalho foi comparar a quantidade e a qualidade de óleo extraído por prensa das duas espécies de andiroba com diferentes teores de umidade e temperatura de secagem das sementes. $O$ delineamento experimental utilizado foi o inteiramente casualizado com quatro repetições em esquema fatorial triplo $(2 \times 3 \times 3)$, sendo duas espécies de andiroba (C. surinamensis e $C$. guianensis), três temperaturas $\left(40,60\right.$ e $\left.80^{\circ} \mathrm{C}\right)$ e três teores de umidade (6, 8 e 10\%), avaliando a quantidade e qualidade do óleo extraível. Após a secagem, as cascas das sementes foram retiradas com auxílio de um martelo e as amêndoas foram trituradas e prensadas a frio em uma prensa hidráulica. Foram analisados os índices de acidez e peróxido dos óleos e comparados a Resolução ANVISA n. 270 de 2005 para óleos brutos prensados a frio. Neste estudo as amêndoas da C. surinamensis liberaram maior quantidade de óleo após a prensagem, independente da umidade e temperatura. O índice de acidez da C. surinamensis (3,37 a 12,52 $\left.\mathrm{mg} \mathrm{KOH} \mathrm{g}^{-1}\right)$ foi maior comparado a $C$. guianensis $\left(2,14\right.$ a 4,74 $\left.\mathrm{mg} \mathrm{KOH} \mathrm{g}^{-1}\right)$. O aumento da temperatura e diminuição do teor de água afetaram a quantidade e qualidade do óleo extraível das sementes de duas espécies de andiroba. Os óleos extraídos das sementes da C. guianensis independentes da temperatura de secagem tiveram os índices de acidez e peróxido de acordo com as normas para óleos brutos prensados a frio. A maior quantidade de óleo extraível das duas espécies de andiroba foi obtida quando a secagem das amêndoas foi feita a $40^{\circ} \mathrm{C}$ com $10 \%$ de umidade.
\end{abstract}

Palavras-chave: Carapa surinamensis; Carapa guianensis; uso múltiplo.

\begin{abstract}
The seeds of andiroba (Carapa surinamensis and Carapa guianensis) were collected on private farms in a rural land environment in Rondônia, Brazil. The objective of this work was to compare the quantity and quality of oil extracted by press of the two species of andiroba with different moisture contents and drying temperature of the seeds. The experimental design was a completely randomized design with four
\end{abstract}

Fonte de financiamento: Nenhuma.

Conflito de interesse: Nada a declarar.

Autor correspondente: jhonatascortes@hotmail.com

Recebido: 25 julho 2018

Aceito: 28 maio 2019.

Editor: Francides Gomes da Silva Júnior.

cc (i) Este é um artigo publicado em acesso aberto (Open Access) sob a licença Creative Commons Attribution, que permite uso, distribuição e reprodução em qualquer meio, sem restrições desde que o trabalho original seja corretamente citado. 
replications in a triple factorial scheme $(2 \times 3 \times 3)$, two species of andiroba (C. surinamensis and C. guianensis), three temperatures $\left(40,60\right.$ and $\left.80^{\circ} \mathrm{C}\right)$ and three $(6,8$ and $10 \%)$, evaluating the quantity and quality of the extractable oil. After drying, the seed husks were removed with the aid of a hammer and the almonds were crushed and cold pressed in a hydraulic press. The acid and peroxide indexes of the oils were analyzed and compared to ANVISA Resolution $n^{\circ} .270$ of 2005 for crude pressed cold oils. In this study almonds from $C$. surinamensis released more oil after pressing, regardless of humidity and temperature. The acid index of $C$. surinamensis (3.37 to $12.52 \mathrm{mg} \mathrm{KOH} \mathrm{g}^{-1}$ ) was higher compared to C. guianensis ( 2.14 to $4.74 \mathrm{mg} \mathrm{KOH} \mathrm{g}^{-1}$ ). The increase in temperature and decrease in water content affected the quantity and quality of extractable oil of the seeds of two andiroba species. The oils extracted from C. guianensis seeds independent of the drying temperature had the acid and peroxide indices according to the norms for cold pressed crude oils. The highest extractable oil content of the two andiroba species was obtained when drying the almonds at $40^{\circ} \mathrm{C}$ at $10 \%$ moisture.

Keywords: Carapa surinamensis; Carapa guianensis; multiple use.

\section{INTRODUÇÃO}

O gênero Carapa, pertence à família das Meliaceae, apresenta o mesmo nome vulgar "andiroba" para as três espécies botânicas: Carapa surinamensis Miq, Carapa vasquezii Kenfack e Carapa guianensis Aubl. O óleo extraído de suas sementes é bastante utilizado na indústria de cosmético e fitoterápico podendo ainda ser utilizado para produção de biodiesel (Mendonça \& Ferraz, 2007; Kenfack, 2011).

A quantidade de óleo extraível das sementes de andiroba varia, de acordo com a espécie, fatores genéticos, ambientais e ainda pelo método de extração adotado (Pantoja, 2007; Queiroz, 2007; Mendonça \& Ferraz, 2007). Em escala experimental a C. surinamensis mostrou maior rendimento de óleo (cerca de $8 \mathrm{~kg}$ de sementes/litro de óleo) do que a C. guianensis (cerca de $11 \mathrm{~kg} / \mathrm{l}$ ) pelo método artesanal (Mendonça \& Ferraz, 2007). Enquanto, no processo de extração por prensa hidráulica foram necessários $2,7 \mathrm{~kg}$ de sementes da $C$. surinamensis e $3 \mathrm{~kg}$ da C.guianensis para obter 1 litro de óleo (Mendonça, 2015).

No processo de extração de óleos vegetais, dois tipos de prensa são utilizados: prensa hidráulica e prensa de rosca (mecânica). A eficiência das prensas de pequena capacidade pode ser aumentada a partir da otimização das principais variáveis envolvidas no processo: pressão aplicada sobre a massa das amêndoas e prévio preparo da matéria-prima a ser processada como: temperatura de prensagem e teor de umidade das sementes (Wiesenborn et al., 2001; Savoire et al., 2013; Rostami et al., 2014).

Ressalta-se ainda que a característica físico-química do óleo, representados pelos índices de acidez e peróxidos, que são os parâmetros referenciais de qualidade e conservação dos óleos vegetais (Brasil, 2005), seja o fator limitante para seu uso. Assim, se fazem necessários procedimentos adequados de secagem das sementes, combinado aos parâmetros mecânicos da prensa.

O conhecimento sobre extração do óleo de andiroba por meio de diferentes métodos ainda é incipiente. Pouco se sabe, por exemplo, sobre a influência da secagem das sementes de andiroba sobre a quantidade e a qualidade do óleo extraível. Diante do exposto, o objetivo do trabalho foi comparar a quantidade e a qualidade de óleo extraído por prensa de duas espécies de andiroba (C. surinamensis e $C$. guianensis) com diferentes teores de umidade e temperatura de secagem das sementes.

\section{MATERIAL E MÉTODOS}

\section{Coleta e tratamento das sementes antes da extração do óleo de andiroba}

As sementes de andiroba foram coletadas em plantios de propriedades particulares em ambiente de terra firme, na zona rural no município do Vale do Paraíso e na linha 04 do município de Ouro Preto d' Oeste (latitude: $10^{\circ} 47^{\prime} 22^{\prime \prime} S$ e longitude: $62^{\circ} 10^{\prime} 22^{\prime \prime}$ W), Rondônia, no período de março a maio de 2014.

As identificações das espécies em estudo foram feitas no Herbário do Instituto Nacional de Pesquisas da Amazônia - INPA em Manaus, e todo o material coletado foi depositado no mesmo Herbário. 
Após a coleta, as sementes boas e inteiras foram separadas das murchas, leves, roídas e escuras. As sementes boas foram mantidas imersas em água por $24 \mathrm{~h}$, a fim de matar, por afogamento, as larvas dos insetos lepidópteros, do gênero Hypsipyla conhecidos como brocadas-sementes da andiroba (Ferraz et al. 2002; Lira-Guedes \& Nardi, 2015) bem como homogeneizar o teor de água de todas as sementes. Em seguida, o teor umidade inicial das sementes foi determinado pelo método da estufa à temperatura de $105^{\circ} \mathrm{C}$ por $24 \mathrm{~h}$ (Brasil, 2009) para então, constituir lotes de $1 \mathrm{~kg}$, em redes de nylon para iniciar o experimento.

O delineamento experimental utilizado foi o inteiramente casualizado com quatro repetições em esquema fatorial triplo $(2 \times 3 \times 3)$, sendo duas espécies de andiroba (Carapa surinamensis e Carapa guianensis), três temperaturas $\left(40,60\right.$ e $\left.80^{\circ} \mathrm{C}\right)$ e três teores de umidade (6, 8 e 10\%), avaliando a quantidade e qualidade do óleo extraível.

Para que as sementes se apresentassem com teores de umidade de: 10,8 e 6\%, antes da extração do óleo, foram levadas à estufa de ventilação forçada nas temperaturas de 40,60 e $80^{\circ} \mathrm{C}$, constituindo os seguintes tratamentos (Tabela 1).

TABELA 1: Tratamentos utilizados no processo de secagem e extração do óleo de sementes de duas espécies de andiroba (C. guianensis e C. surinamensis).

\begin{tabular}{ccc}
\hline Temperatura $\left({ }^{\circ} \mathrm{C}\right)$ & Teor de umidade (\%) \\
\hline & & 10 \\
40 & C. surinamensis & 8 \\
& & 6 \\
& & 10 \\
& C. guianensis & 8 \\
& & 6 \\
60 & C. surinamensis & 10 \\
& & 8 \\
& & 6 \\
& C. guianensis & 10 \\
& & 8 \\
& & 6 \\
& C. surinamensis & 10 \\
& & 8 \\
& & 6 \\
& C. guianensis & 10 \\
& & 8 \\
\hline
\end{tabular}

Para cada tratamento foram usados oito quilos de sementes e quatro repetições. A secagem foi determinada por meio da Equação (1) proposta por Almeida et al. (2006).

$$
\mathrm{mf}=\frac{\mathrm{mi}^{*}(100-\mathrm{Ui})}{(100-\mathrm{Uf})}
$$

Em que: $\mathrm{mf}=$ massa final; $\mathrm{mi}=$ massa inicial; $\mathrm{Ui}=$ teor de umidade inicial; Uf $=$ teor de umidade final.

\section{Quantidade do óleo extraído por prensa hidráulica}

As sementes após atingirem o teor de umidade final, tiveram as cascas retiradas com auxílio de um martelo e as amêndoas foram trituradas com auxílio de moinho de facas e prensadas em uma prensa hidráulica a frio confeccionada por Andreza Mendonça para uso experimental em laboratório, com tambor de inox, com pressão inicial de 3 toneladas e final de 15 toneladas, por um período de $4 \mathrm{~h}$. Ao final da extração, o óleo filtrado em papel filtro e transferido de um recipiente âmbar para uma proveta graduada em $\mathrm{mL}$. 


\section{Qualidade dos óleos extraídos por prensa hidráulica}

A qualidade dos óleos de andiroba brutos prensados a frio foi determinada a partir da análise dos índices de acidez, peróxido, iodo e saponificação de acordo com a metodologia descrita pelo Instituto Adolfo Lutz (2008). Cada amostra de óleo extraído foi avaliada em triplicata.

A qualidade dos óleos extraídos foi avaliada por meio dos valores médios obtidos nas análises por tratamento e comparado com os índices determinados pela Resolução da Agência Nacional de Vigilância Sanitária (ANVISA) nº 270 para óleos brutos prensados a frio (Brasil, 2005).

\section{Análise estatística}

Os dados quantitativos foram submetidos à análise de variância pelo teste de $F$, a 0,05 de probabilidade, e quando significativas, as médias foram comparadas pelo teste de Tukey a 0,05 de probabilidade. O software utilizado foi o Sisvar versão 5,6 (build 86) (Ferreira, 2011).

\section{RESULTADOS E DISCUSSÃO}

\section{Quantidade de óleo extraível por prensa hidráulica}

A quantidade de óleo extraível para as duas espécies de andiroba apresentaram diferença significativa em relação às espécies e às temperaturas de secagem das sementes (Tabela 2). Verificou-se também interação significativa entre as temperaturas e umidades das sementes para as duas espécies testadas (Tabela 2).

TABELA 2: Análise de variância dos valores médios da quantidade de óleo extraível $(\mathrm{mL})$ de $1 \mathrm{~kg}$ de amêndoas sem casca de duas espécies de andiroba (Carapa surinamensis e Carapa guianensis), submetidas a diferentes temperaturas de secagem e teores de umidade.

\begin{tabular}{|c|c|c|}
\hline \multirow{2}{*}{ Fontes de variação } & \multirow{2}{*}{ GL } & \multirow{2}{*}{$\begin{array}{c}\text { F calculado } \\
\text { Óleo }\end{array}$} \\
\hline & & \\
\hline Espécies (E) & 1 & $83,723 * *$ \\
\hline Temperatura (T) & 2 & $87,249 * *$ \\
\hline Umidade (U) & 2 & $0,829^{\text {ns }}$ \\
\hline$E \times T$ & 2 & $83,850 * \star$ \\
\hline$E \times U$ & 2 & $7,797 * \star$ \\
\hline$T \times U$ & 4 & $20,490 * *$ \\
\hline$E \times T \times U$ & 4 & $8,706 * *$ \\
\hline Erro & 54 & 589,157 \\
\hline $\mathrm{CV} \%$ & - & 8,61 \\
\hline
\end{tabular}

Em que: $G \mathrm{~L}$ = graus de liberdade; $\mathrm{CV}=$ coeficiente de variação; ** $=(p<0,01) ; \mathrm{ns}=$ não significativo.

O aumento do teor de água das amêndoas submetidas à secagem em baixa temperatura contribuiu para maior liberação de óleo, contudo o comportamento foi inverso quando as sementes foram secas em $80^{\circ} \mathrm{C}$ para as duas espécies (Tabela 3). Neste estudo as amêndoas da Carapa surinamensis liberaram maior quantidade de óleo após a prensagem, independente da umidade e temperatura, com exceção as sementes secas a $60^{\circ} \mathrm{C}$ onde não houve diferença entre as espécies (Tabela 3 e 4). 
TABELA 3: Valores médios da interação entre duas espécies de andiroba (Carapa surinamensis and Carapa guianensis) e diferentes temperaturas de secagem e teores de umidade sob quantidade de óleo extraível $(\mathrm{mL})$ a partir da prensagem de $1 \mathrm{~kg}$ de amêndoas sem casca.

\begin{tabular}{|c|c|}
\hline \multirow{3}{*}{$\begin{array}{c}\text { Temperatura } \\
\left({ }^{\circ} \mathrm{C}\right)\end{array}$} & Carapa surinamensis \\
\hline & Umidade (\%) (b.u.) \\
\hline & 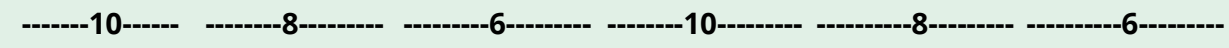 \\
\hline 40 & A $368,25 X$ a A $367,75 X$ a A $360,75 X$ a A $329,75 Y$ a $A 313,25 Y$ a $B 264,25 Y$ a \\
\hline 60 & A 305,50 X b C 191,25 Y b B 236,25 X b A 302,25 X a A $302,75 X$ a B $254,00 X$ a \\
\hline 80 & B $239,00 X$ с A 346,50 X a A 360,25 X a C 160,75 Y b C 185,25 Y b C 183,75 Y b \\
\hline
\end{tabular}

Médias antecedidas de mesma letra maiúscula ( $A, B$ e $C$ ) na linha (para umidade) e seguidas de mesma letra minúscula ( $a$, b e c) na coluna (para temperaturas de secagem) e maiúscula ( $X$ e $Y$ ) na linha (para espécies) não diferem entre si pelo teste de Tukey, a $0,05 \%$ de probabilidade.

TABELA 4: Quantidade de óleo extraível $(\mathrm{mL})$ de duas espécies de andiroba (Carapa surinamensis e Carapa guianensis), submetidas a diferentes temperaturas de secagem e teores de umidade.

\begin{tabular}{|c|c|c|c|c|c|c|c|c|c|}
\hline \multirow{3}{*}{$\begin{array}{c}\text { Temperatura }\left({ }^{\circ} \mathrm{C}\right) \\
40\end{array}$} & \multicolumn{9}{|c|}{ Carapa surinamensis } \\
\hline & \multicolumn{3}{|c|}{--------10-------- } & \multicolumn{3}{|c|}{-----------8---------- } & \multicolumn{3}{|c|}{---------6--------- } \\
\hline & 368,25 & $a$ & $A$ & 367,75 & $a$ & A & 360,75 & $a$ & A \\
\hline 60 & 305,50 & b & $A$ & 191,25 & $b$ & B & 236,25 & b & B \\
\hline \multirow[t]{2}{*}{80} & 239,00 & c & $B$ & 346,50 & a & A & 360,25 & a & A \\
\hline & \multicolumn{8}{|c|}{ Carapa guianensis } & \\
\hline 40 & 329,75 & a & $A$ & 313,25 & a & A & 264,25 & a & B \\
\hline 60 & 302,25 & b & $A$ & 302,75 & a & A & 254,00 & a & B \\
\hline 80 & 160,75 & c & $B$ & 185,25 & b & A & 183,75 & $b$ & A \\
\hline
\end{tabular}

Médias de mesma letra minúscula ( $a, b$ e $c$ ) na coluna (para temperatura) e seguidas de mesma letra maiúscula ( $A, B$ e C) na linha (para umidade) não diferem dentro de cada espécie entre si pelo teste de Tukey, a 0,05\% de probabilidade.

As sementes grandes ovais ou esféricas são geralmente sensíveis a dessecação, o fato de serem volumosas reduz a velocidade de perda de água (Tweddle et al., 2003), o que justifica serem secas sob baixas temperaturas $\left(40\right.$ a $60{ }^{\circ} \mathrm{C}$ ). Pighinelli et al. (2008) ao estudarem otimização da prensagem a frio de grãos de amendoim em prensa tipo expeller, verificaram que o maior rendimento de óleo foi para temperaturas entre 50 e $65^{\circ} \mathrm{C}$ e teor de umidade entre 10 e 10,8\%, semelhante a este experimento.

Verificou-se também que a temperatura de secagem influenciou na quantidade de óleo extraível para as duas espécies, com maior liberação de óleo das sementes que foram secas a $40{ }^{\circ} \mathrm{C}$ e a $10 \%$ de umidade (Tabela 4 ).

Desse modo, pode-se verificar também que secando as sementes a $40{ }^{\circ} \mathrm{C}$ até $10 \%$ de umidade seriam necessários 2,7 kg de amêndoas da Carapa surinamensis para extrair um litro de óleo e 3,0 kg da Carapa guianensis (Tabela 4). Deve-se salientar que no experimento foram utilizadas apenas amêndoas sem cascas e a extração ocorreu em prensa hidráulica, possivelmente o manejo das sementes combinado à extração em prensa mecânica tipo expeller teria aumentado a quantidade de óleo extraível.

A quantidade de óleo extraível foi maior nesse experimento do que no trabalho de Mellinger (2006) na comunidade Boa Esperança, Amazonas, em que foram usados 4,55 kg de sementes Carapa guianensis com casca para produzir $1 \mathrm{~L}$ de óleo por meio de prensa expeller.

No Amapá, Gomes (2010) comparou a extração do óleo por meio de prensa mecânica de 9 toneladas usando sementes da Carapa guianensis secas a $70{ }^{\circ} \mathrm{C}$ até peso constante provenientes de floresta de terra firme e de várzea. A maior quantidade de óleo foi obtida das sementes sem casca da floresta de várzea (203,5 L de óleo para cada $1000 \mathrm{~kg}$ de sementes, ou seja, 4,9 kg de sementes para cada litro de óleo) em relação à terra firme $(158,6 \mathrm{~L}$ de óleo para $1000 \mathrm{~kg}$ de sementes, o que equivale a $5,8 \mathrm{~kg}$ de sementes para cada litro de óleo). 
Nas usinas de extração de óleo do Amazonas e Rondônia, os cooperados declararam que são necessários de 3,3 a 6 kg de sementes para obter um litro de óleo (Mendonça, 2015). A autora ressaltou que as usinas processam as duas espécies de andiroba misturadas e a prensagem é realizada com as sementes trituradas com casca e não há controle do teor de umidade das sementes antes da prensagem.

A mistura das duas espécies de andiroba e o teor de água das sementes abaixo de 10\% assim como a prensagem das sementes com casca, possivelmente explique porque mesmo após duas prensagens, raramente as indústrias na Amazônia exceda 30\% de rendimento de óleo (Pinto, 1956; Mendonça, 2015).

\section{Qualidade do óleo extraído por prensa hidráulica}

Os óleos da Carapa guianensis, em quase todos os tratamentos, tiveram índices de acidez menores do que o valor estabelecido pela Resolução ANVISA n. 270 (Brasil, 2005) para óleos brutos prensados a frio $\left(4,0 \mathrm{mg} \mathrm{KOH} \mathrm{g}^{-1}\right)$ (Tabela 5).

TABELA 5: Qualidade do óleo de andiroba extraível a partir da prensagem de $1 \mathrm{~kg}$ de amêndoas em prensa hidráulica de 15 t em função da temperatura e umidade das amêndoas de duas espécies.

\begin{tabular}{|c|c|c|c|c|c|}
\hline \multirow[b]{2}{*}{$\begin{array}{c}\text { Temperatura } \\
\left({ }^{\circ} \mathrm{C}\right)\end{array}$} & \multicolumn{5}{|c|}{ Carapa surinamensis } \\
\hline & Umidade (\%) & 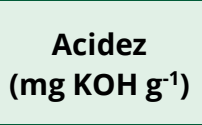 & $\begin{array}{c}\text { Peróxido } \\
\text { (meq kg }{ }^{-1} \text { de } \\
\text { óleo) }\end{array}$ & $\begin{array}{c}\text { lodo } \\
\left(\mathrm{mg} \mathrm{I}_{2} 100 \mathrm{~g}^{-1}\right)\end{array}$ & $\begin{array}{c}\text { Saponificação } \\
\left(\mathrm{mg} \mathrm{KOH} \mathrm{g}^{-1}\right)\end{array}$ \\
\hline \multirow{4}{*}{40} & 6 & 4,48 & 0,32 & 74,08 & 215,83 \\
\hline & 8 & 5,03 & 0,53 & 83,88 & 204,99 \\
\hline & 10 & 5,39 & 1,12 & 80,56 & 206,14 \\
\hline & 6 & 5,67 & 0,88 & 76,16 & 209,00 \\
\hline \multirow[t]{3}{*}{60} & 8 & 3,37 & 0,59 & 72,11 & 210,11 \\
\hline & 10 & 4,49 & 1,44 & 75,81 & 211,97 \\
\hline & 6 & 8,13 & 2,44 & 75,03 & 218,54 \\
\hline \multirow[t]{4}{*}{80} & 8 & 10,22 & 0,79 & 72,56 & 210,14 \\
\hline & 10 & 12,52 & 0,61 & 69,66 & 184,90 \\
\hline & \multicolumn{5}{|c|}{ Carapa guianensis } \\
\hline & 6 & 2,24 & 0,46 & 70,39 & 163,13 \\
\hline \multirow[t]{3}{*}{40} & 8 & 3,05 & 0,74 & 76,26 & 208,74 \\
\hline & 10 & 3,60 & 0,85 & 72,59 & 202,69 \\
\hline & 6 & 4,00 & 0,34 & 63,89 & 209,86 \\
\hline \multirow[t]{3}{*}{60} & 8 & 4,74 & 0,30 & 59,03 & 211,02 \\
\hline & 10 & 3,61 & 0,15 & 63,74 & 208,31 \\
\hline & 6 & 2,14 & 2,26 & 67,53 & 192,03 \\
\hline \multirow[t]{2}{*}{80} & 8 & 2,27 & 2,28 & 67,00 & 192,44 \\
\hline & 10 & 4,30 & 2,26 & 65,41 & 198,01 \\
\hline
\end{tabular}

Verificou-se também que o aumento da temperatura de secagem das sementes alterou a coloração do óleo, sendo mais evidente nos óleos da Carapa surinamensis, do que nos da Carapa guianensis. Deve-se considerar que as sementes da Carapa surinamensis são menores e tem casca menos espessa do que a Carapa guianensis (Mendonça et al., 2015), consequentemente, secam mais rápido.

A temperatura de secagem das sementes é um dos fatores mais importantes quando se trata de qualidade de óleos, podendo afetar suas propriedades físico-químicas, levar à rancificação de gorduras e alterar pigmentos quando submetidos a altas temperaturas. Dessa forma, a seleção das condições de operação (tipo de secagem e tempo) que minimizam essas alterações é importante para obtenção de produtos de qualidade (Aquino et al., 2009).

Quanto à oxidação do óleo, o índice de peróxido dos óleos das duas espécies de andiroba foram menores do que o valor máximo estabelecido pela Resolução ANVISA n. 270, que é de 


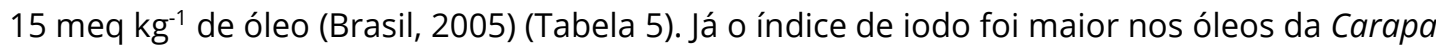
surinamensis. Isso deve ter ocorrido, devido ao maior grau de insaturação das cadeias carbônicas (Ramalho et al., 2011). Verificou-se também que os maiores índices de iodo para as duas espécies foram a temperatura de $40^{\circ} \mathrm{C}$ em relação as demais temperaturas testadas, os altos valores podem ser explicados pela maior quantidade de ácidos graxos poliinsaturados. O índice de saponificação da Carapa surinamensis foi maior do que na Carapa guianensis (Tabela 5).

Estudo realizado por Vasconcelos et al. (2009) testaram diferentes métodos de extração do óleo de andiroba (Carapa guianensis): prensagem das sementes com 5\% de umidade e acondicionada a $70{ }^{\circ} \mathrm{C}$ por $3 \mathrm{~h}$, extração artesanal, extração por solvente das sementes in natura e fermentadas. Os autores registraram menor rendimento no processo de prensagem com sementes com $5 \%$ de umidade $(12,29 \%)$ e maior rendimento por meio do processo de extração por solvente com sementes fermentadas (29,74\%).

Os valores dos índices de acidez (8,82 e 10,66 $\mathrm{mg} \mathrm{KOH} \mathrm{g}^{-1}$ ) e peróxidos (8,38 e 7,17 meq kg-1) registrados por Vasconcelos et al (2009) foram superiores aos recomendados pela Resolução ANVISA n. 270 (Brasil, 2005).

O óleo de andiroba é constituído basicamente de ácidos graxos. O perfil de ácidos graxos tem forte relação com a oxidação lipídica, pois quanto maior a quantidade de ácidos graxos insaturados, maior a possibilidade de degradação oxidativa (Krichene et al., 2010; Silva et al., 2010). Desse modo, fica evidente que os tratamentos de secagem combinado ao método de extração influenciam diretamente na quantidade e qualidade do óleo.

\section{CONCLUSÃO}

A quantidade de óleo extraído das sementes de Carapa surinamensis é maior do que da Carapa guianensis independente da temperatura e umidade de secagem.

A maior quantidade de óleo extraível das duas espécies de andiroba (C. surinamensis e C. guianensis) foi obtida com a secagem das amêndoas a $40{ }^{\circ} \mathrm{C}$ a $10 \%$ de umidade.

Os óleos extraídos das sementes da C. guianensis independentes da temperatura de secagem e tiveram os índices de acidez e peróxido de acordo com as normas para óleos brutos prensados a frio

\section{AGRADECIMENTOS}

Agradecemos ao Instituto Federal de Rondônia campus Ji-Paraná, por meio do edital de pesquisa n. 03 de 2013, ao Núcleo de Estudos em Agroecologia e ao CNPq por meio do edital $\mathrm{n}^{\circ} 058$ de 2010.

\section{REFERÊNCIAS}

Almeida, F. A. C., Duarte, M. E. M., \& Mata, M. E. R. M. C. (2006). Tecnologia de armazenamento em sementes (402 p.). Campina Grande: UFCG.

Aquino, L. P., Ferrua, F. Q., Borges, S. V., Antoniassi, R., Correa, J. L. G., \& Cirillo, M. A. (2009). Influência da secagem do pequi (Caryocar brasiliensis Camb.) na qualidade do óleo extraído. Food Science and Technology (Campinas), 29(2), 354-357. http://dx.doi.org/10.1590/S0101-20612009000200018.

Brasil. Agência Nacional de Vigilância Sanitária - ANVISA. (2005, 23 de setembro). Aprova o "Regulamento Técnico" para Óleos vegetais, Gorduras Vegetais e Creme vegetal", constante do Anexo desta Resolução (Resolução RDC n²70, de 22 de setembro de 2005). Diário Oficial [da] República Federativa do Brasil, Brasília.

Brasil. Ministério da Agricultura e Reforma Agrária. Secretaria Nacional de defesa Agropecuária. (2009). Regras para análise de sementes (365p). Brasília: MAPA.

Ferraz, I. D. K., Camargo, J. L. C.; Sampaio, P. T. B. (2002). Sementes e plântulas de andiroba (Carapa guianensis Aubl. e Carapa procera D. C.): Aspectos botânicos, ecológicos e tecnológicos. Acta amazônica, 32(4), 647-661.

Ferreira, D. F. (2011). Sisvar: a computer statistical analysis system. Ciência e Agrotecnologia, 35(6), 1039 1042. http://dx.doi.org/10.1590/S1413-70542011000600001. 
Gomes, H. S. R. (2010). Estrutura populacional e produção de andiroba em terra firme e várzea no sul do Amapá (Dissertação de mestrado). Universidade Federal do Amapá, Macapá.

Instituto Adolfo Lutz - IAL. (2008). Métodos físico-químicos para análise de alimentos (4. ed., 1020 p.). São Paulo: Instituto Adolfo Lutz.

Kenfack, D. (2011). Resurrection in Carapa (Meliaceae): A reassessment of morphological variation and species boundaries using multivariate methods in a phylogenetic context. Botanical Journal of the Linnean Society, 165(2), 186-221. http://dx.doi.org/10.1111/j.1095-8339.2010.01104.x.

Krichene, D., Allalout, A., Mancebo-Campos, V., Salvador, M. D., Zarrouk, M., \& Fregapane, G. (2010). Stability of virgin olive oil and behavior of its natural antioxidants under medium temperature accelerated storage conditions. Food Chemistry, 121(1), 171-177. http://dx.doi.org/10.1016/j.foodchem.2009.12.026.

Lira-Guedes, A. C., \& Nardi, M. (2015). Guia prático para o manejo sustentável de andirobeiras de várzea e para a extração do óleo de suas sementes (24p). Brasília: Embrapa.

Mellinger, L. L. (2006). Aspectos da regeneração natural e produção de sementes de Carapa guianensis Aubl. (andiroba) na Reserva de Desenvolvimento Sustentável Amanã (AM) (Dissertação de mestrado). Universidade Federal do Amazonas, Manaus.

Mendonça, A. P. (2015). Secagem e extração do óleo das sementes de andiroba (Carapa surinamensis Miq. e Carapa guianensis Aubl.) (Tese de doutorado). Instituto Nacional de Pesquisas da Amazônia, Manaus.

Mendonça, A. P., \& Ferraz, I. D. K. (2007). Óleo de andiroba: processo tradicional da extração, usos e aspectos sociais no estado do Amazonas, Brasil. Acta Amazonica, 37(3), 353-364. http://dx.doi.org/10.1590/S0044-59672007000300006.

Mendonça, A. P., Sampaio, P. T. B., Almeida, F. A. C., Ferreira, R. F., \& Novais, J. M. (2015). Determinação das curvas de secagem das sementes de andiroba em secador solar. Revista Brasileira de Engenharia Agrícola e Ambiental, 19(4), 382-387. https://doi.org/10.1590/1807-1929/agriambi.v19n4p382-387.

Pantoja, T. F. (2007). Descrição morfológica e análise da variabilidade genética para caracteres de frutos, sementes e processo germinativo associado à produtividade de óleo em matrizes de Carapa guianensis Aublet., uma Meliaceae da amazônia (Dissertação de mestrado). Universidade Estadual Paulista, Jaboticabal.

Pighinelli, A. L. M. T., Park, K. J., Rauen, A. M., Bevilaqua, G., \& Guillaumon Filho, J. A. (2008). Otimização da prensagem a frio de grãos de amendoim em prensa tipo expeller. Food Science and Technology (Campinas), 28, 66-71. http://dx.doi.org/10.1590/S0101-20612008000500011.

Pinto, G. P. (1956). Contribuição ao estudo químico do óleo de andiroba. Boletim Técnico do Instituto Agronômico do Norte, 31, 195-206.

Queiroz, J. A. L. D. (2007). Guia prático de manejo florestal para produção de frutos de andiroba (Carapa guianensis Aubl.) e outros produtos de valor econômico no estado do Amapá: a floresta pode dar bons frutos (38 p.). Macapá: IEPA.

Ramalho, E. F. S. M., Albuquerque, A. R., Souza, A. L., Barro, A. K., Maia, A. S., Santos, I. M. G., \& Souza, A. G. (2011). Use of different techniques in the evalution of the oxidative stability of poultry fat biodiesel. Journal of Thermal Analysis and Calorimetry, 106(3), 787-791. http://dx.doi.org/10.1007/s10973-011-1545-2.

Rostami, M., Farzaneh, V., Boujmehrani, A., Mohammadi, M., \& Bakhshabadi, H. (2014). Optimizing the extraction process of sesame seed's oil using response surface method on the industrial scale. Industrial Crops and Products, 58, 160-165. http://dx.doi.org/10.1016/j.indcrop.2014.04.015.

Savoire, R., Lanoisellé, J. L., \& Vorobiev, E. (2013). Mechanical continuous oil expression from oilseeds: a review. Food and Bioprocess Technology, 6(1), 1-16. http://dx.doi.org/10.1007/s11947-012-0947-x.

Silva, L., Pinto, J., Carrola, J., \& Paiva-Martins, F. (2010). Oxidative stability of oil after food processing and comparison with other vegetable oils. Food Chemistry, 121(4), 1177-1187. http://dx.doi.org/10.1016/j.foodchem.2010.02.001.

Tweddle, J. C., Dickie, J. B., Baskin, C. C., \& Baskin, J. M. (2003). Ecology of seed desiccation sensitivity. Journal of Ecology, 91(2), 294-304. http://dx.doi.org/10.1046/j.1365-2745.2003.00760.x.

Vasconcelos, M. A. M., Mattietto, R. A., Gonçalves, A. C. S., Oliveira, P. S., Moreira, P. I. O., Alves, S. M., Moreira, D. K. T., Figueiredo, J. G., \& Dantas Filho, H. A. (2009). Avaliação do processo de extração e caracterização do óleo e sementes de andiroba (Carapa guianensis Aublet). In Anais da $7^{\circ}$ Conferência do Subprograma de Ciência e Tecnologia - SPC\&T FASE II/PPG (pp. 365-367). Brasília: CNPq. CD-ROM. 
Wiesenborn, D., Doddapaneni, R., Tostenson, K., \& Kangas, N. (2001). Cooking indices to predict screwpress performance for crambe seed. Journal of the American Oil Chemists' Society, 78(5), 467-471. http://dx.doi.org/10.1007/s11746-001-0287-y.

Contribuição dos Autores: APM: conceituação, curadoria de dados, investigação, metodologia, escrita - primeira redação, escrita - revisão e edição; FACA: conceituação, metodologia; ASO: investigação; JCR: análise formal, escrita - revisão e edição; MERA: análise formal, metodologia; PTBS: conceituação, metodologia. 